\title{
A Matrix Problem Concerning Projections
}

\author{
By Hans Schneider \\ (Received 1sth February 1953).
}

The following problem is a slight generalisation of one posed and partly solved by $H$. Nagler. ${ }^{1}$ We shall use $A^{*}$ for the conjugate transpose of a matrix $A$. A projection is an idempotent matrix, its latent roots consist of units and zeros.

Problem. Let $A$ be an $n \times m$ matrix with complex elements. Find an $m \times n$ matrix $B$ such that $(I-A B)^{*}(I-A B)$ is a projection of rank $k$.

Let the rank of $A$ be $r$. The rank of $A B$ is less than or equal to $r$. It is easily proved that the rank of $M=I-A B$ cannot be less than $n-r$. But the rank of $M^{*} M$ equals that of $M$, and hence

$$
k \geqslant n-r
$$

is a necessary condition for the existence of a matrix $B$ with the required property.

We shall next show that ( 1 ) is also a sufficient condition. It is enough to find a matrix $B$ such that $I-A B$ is a Hermitian projection of rank $k$.

The $n \times n$ matrix $A A^{*}$ is Hermitian of rank $r$. Hence there exist $n$ Hermitian projections of rank 1 satisfying

$$
\begin{aligned}
\sum_{1}^{n} E_{i} & =I, \\
E_{i} E_{j} & =0 \text { when } i \neq j, \\
\sum_{1}^{n} \rho_{i} E_{i} & =A A^{*},
\end{aligned}
$$

where the $\rho_{i}$ are the (non-negative) latent roots of $A A^{*}$, supposed arranged so that $\rho_{i} \neq 0$ for $i=1, \ldots, r$, and $\rho_{i}=0$ for $i=r+1, \ldots, n$.

Let

$$
C=\sum_{1}^{n} \bar{\Sigma}_{i}^{k} E_{i}
$$

where

$$
\sigma_{i}=1 / \rho_{i} \text { for } i=1, \ldots, n-k \leqslant r .
$$

We note that $\quad A A^{*} C=\sum_{1}^{n-k} E_{i}$,

1 H. Nagler, "On a certain matrix pruduct, with specified latent roots," Proc. Edinburgh Math. Soc. (2), 10 (1953), 21-24. 
whence it follows that

$$
I-A A^{*} C=\sum_{n-k+1}^{n} E_{i}
$$

is a Hermitian projection of $\operatorname{rank} k$. Thus $B=A^{*} C$ is a matrix having the desired property.

Let $x_{1}, \ldots, x_{n}$ form an orthonormal set of latent column vectors of $A A^{*}$, where $x_{i}$ is associated with the latent root $\rho_{i}$. It may be romarked that a set of Hermitian projections of rank 1 satisfying (2), (3) and (4) is given by $E_{i}=x_{i} x_{i}^{*}$ for $i=1, \ldots, n$.

Suppose now that $k=n-r$. When $m \leqslant n$ and the rank of $A$ is $m$, then we assert that our method yields $B=\left(A^{*} A\right)^{-1} A^{*}$, while if $n \leqslant m$ and the rank of $A$ is $n$, then $B=A^{*}\left(A A^{*}\right)^{-1}$. The proof of this is left to the reader. In general, it is clear from the method of construction that the solution we have found is not unique.

An $m \times n$ matrix $D$ for which $I-D A$ is a Hermitian projection of rank $k$ can be found in a similar manner, provided that $k \geqq m-r$.

The Queen's University,

BELFAST. 